\title{
Post Disaster Mapping with Semantic Change Detection in Satellite Imagery
}

\author{
Ananya Gupta, Elisabeth Welburn, Simon Watson and Hujun Yin \\ School of Electrical and Electronic Engineering \\ University of Manchester \\ ananya.gupta@manchester.ac.uk
}

\begin{abstract}
Accurate road maps are important for timely disaster relief efforts and risk management. Current disaster mapping is done manually by volunteers following a disaster and the process is slow and error prone. We propose a framework for identifying accessible roads in post-disaster satellite imagery by detecting changes from pre-disaster imagery, in conjunction with OpenStreetMap data. We validate our results with data from Indonesia 2018 tsunami, obtained from DigitalGlobe.
\end{abstract}

\section{INTRODUCTION}

Globally, hundreds of natural disasters happen every year ${ }^{1}$. Identifying high impact areas and accurate mapping of the post disaster landscape are extremely important for disaster relief and recovery efforts. It would allow for damage assessment and identifying routes for early responses to affected areas.

Currently, following natural disasters that lead to humanitarian crises, this mapping is done by volunteer initiatives around the world using satellite imagery [1]. However, the process is time consuming and also inconsistent across the various initiatives as the volunteers are mostly inexperienced and follow different processes [2].

A possible solution to identify changed areas is to compare pixel values of satellite images before and after the disaster. However, due to a number of factors such as lighting, season and weather, the pixels cannot be compared directly. In order to tackle these challenges, there is a growing trend of using Convolutional Neural Networks (CNNs) to analyse post-disaster imagery for damage assessment in buildings $[3,4,5]$. However, the existing work depends on having a large amount of manually annotated training data, which is either often unavailable or requires timeconsuming and unscalable tasks.

Most of the current approaches for automatic road ex-

\footnotetext{
${ }^{1}$ https://ourworldindata.org/natural-disasters
}

traction treat the problem as a semantic segmentation task where small crops of satellite images are used to predict the probability of a pixel being a road or non-road [6]. However, a major issue with this approach is that roads are often missed due to shadows and occlusions by buildings and trees. A number of post processing techniques have to be used to reason about missing roads. These techniques are based on heuristics and typically reason about shortest paths between broken roads. Though such a method works well in practice for most cities, it is not suitable for post disaster scenarios since the broken connections could actually be missing roads due to the effects of the disaster.

In order to alleviate this issue, we propose a framework to use both pre-event and post-event imagery for change detection in conjunction with the OSM data to obtain an upto-date road map of the affected areas.

\section{METHODOLOGY}

The proposed method consists of a number of steps: 1) a CNN is used to segment satellite images into road and non-road regions; 2) the segmented image is converted into a road network graph; 3 ) the pre and post disaster satellite images are compared to identify the impacted areas and possible missing road segments. This pipeline is visualised in Fig. 1.

Inspired by the family of encoder-decoder segmentation models, we have developed a LinkNet [7] based network for the task of road segmentation. The encoder is based on ResNet34 [8] pretrained on the ImageNet, which has been found to yield good performances for this task without excessive computational costs. The output of each layer in the encoder feeds into the input of the corresponding decoder layer in order to help recover details lost during the convolution due to the downsampling of the input. The network is trained with a binary cross entropy loss function. The output from the segmentation network is used to create a binary road mask, $M$.

We compute the difference in the output masks from predisaster and post-disaster road imagery to find the affected areas. Morphological opening is carried out to remove fur- 
Table 1: Quality, Completeness, Correctness Results

\begin{tabular}{|l|l|l|l|}
\hline Method & Precision & Recall & F-score \\
\hline DRM[6] & 0.86 & 0.93 & 0.89 \\
\hline OSM Diff(Ours) & 0.94 & 0.99 & 0.96 \\
\hline
\end{tabular}

Table 2: Connectivity Results. Correct implies that the shortest paths are similar in length to the ground truth, No Conn is where there was no possible path, Too Short is where the paths were too short compared to the ground truth and Too Long is where the path was too long. All values are given as percentages.

\begin{tabular}{|l|l|l|l|l|}
\hline Graph Type & Correct & $\begin{array}{l}\text { No } \\
\text { Conn }\end{array}$ & $\begin{array}{l}\text { Too } \\
\text { Short }\end{array}$ & $\begin{array}{l}\text { Too } \\
\text { Long }\end{array}$ \\
\hline DRM[6] & 41.31 & 40.17 & 6.21 & 12.26 \\
\hline OSM Diff(Ours) & 73.38 & 16.76 & $\mathbf{2 . 0 3}$ & 7.72 \\
\hline OSM Weighted Diff(Ours) & $\mathbf{8 6 . 5 9}$ & $\mathbf{0}$ & 8.18 & $\mathbf{5 . 1 3}$ \\
\hline
\end{tabular}

ther noise from the image. This final image provides the routes that changed due to the disaster.

The difference mask is converted to a network graph, $G_{\text {diff }}$, by skeletonising the image. The skeleton is then traversed to find nodes in the output graph such that any positive pixel with more than two positive neighbours is assumed to be a junction and all pixels between two junctions are marked as belonging to an edge.

The Ramer-Douglas-Pecker algorithm [9] is used to approximate the edges with piece-wise line segments. Each linear segment is then sliced into smaller sub-segments of a fixed length, $l$. All such segments can then be compared to find corresponding segments in the graphs.

An ideal pre-disaster road network $G_{\text {pre-ideal }}$ for the region is obtained from the publicly available OSM dataset. For each edge in $G_{\text {diff }}$, the closest edge in $G_{\text {pre-ideal }}$ is found and removed.

\section{EXPERIMENTS}

We used imagery from Digital Globe's Open Data Initiative and extracted an area around Palu city that was devastated by a tsunami and earthquake for training and evaluation. We also downloaded the publicly available data from the OSM for this region and extracted all roads.

Our results have been summarised in Table 1 and Table 2 and compared to the baseline [6].

\section{CONCLUSIONS}

In this paper, we have provided a framework for identifying the road topology from satellite images in post-disaster scenarios. The proposed method brings the time required for identifying roads in disaster struck areas down to minutes which will help provide timely assistance.
The proposed method is based on using a CNN for semantic segmentation to identify roads in imagery, which can then be converted to a road network graph. We use publicly available data to train our networks and hence do not require costly annotation processes in order to create training data. The results from pre-disaster and post-disaster imagery are compared to quantify the changes in the environment and used in conjunction with data from the OSM to obtain a more realistic map of the environment.

The proposed approach has shown promising results on a dataset on Palu, Indonesia struck by a tsunami and an earthquake in 2018. A promising direction for future work would be identifying what causes the breaks in roads to get a more realistic map of the environment.

\section{References}

[1] P. Boccardo and F. Giulio Tonolo, "Remote Sensing Role in Emergency Mapping for Disaster Response," in Engineering Geology for Society and Territory - Volume 5, pp. 17-24, Cham: Springer International Publishing, 2015.

[2] T. H. Poiani, R. d. S. Rocha, L. C. Degrossi, and J. P. d. Albuquerque, "Potential of collaborative mapping for disaster relief: A case study of openstreetmap in the Nepal earthquake 2015," in Proceedings of the Annual Hawaii International Conference on System Sciences, vol. 2016-March, pp. 188197, IEEE, 12016.

[3] T. G. J. Rudner, M. Rußwurm, J. Fil, R. Pelich, B. Bischke, V. Kopackova, and P. Bilinski, "Multi3Net: Segmenting Flooded Buildings via Fusion of Multiresolution, Multisensor, and Multitemporal Satellite Imagery," in Thirty-Third AAAI Conference on Artificial Intelligence, 2019.

[4] S. N. K. B. Amit and Y. Aoki, "Disaster detection from aerial imagery with convolutional neural network," in International Electronics Symposium on Knowledge Creation and Intelligent Computing, pp. 239-245, IEEE, 92017.

[5] A. Fujita, K. Sakurada, T. Imaizumi, R. Ito, S. Hikosaka, and R. Nakamura, "Damage detection from aerial images via convolutional neural networks," in 5th IAPR International Conference on Machine Vision Applications, pp. 5-8, IEEE, 5 2017.

[6] G. Mattyus, W. Luo, and R. Urtasun, "DeepRoadMapper: Extracting Road Topology from Aerial Images," in IEEE International Conference on Computer Vision, vol. 2017-Octob, pp. 3458-3466, IEEE, 102017.

[7] A. Chaurasia and E. Culurciello, "LinkNet: Exploiting encoder representations for efficient semantic segmentation," in 2017 IEEE Visual Communications and Image Processing, VCIP 2017, vol. 2018-Janua, pp. 1-4, 62018.

[8] K. He, X. Zhang, S. Ren, and J. Sun, "Deep Residual Learning for Image Recognition," in IEEE Conference on Computer Vision and Pattern Recognition, 2016.

[9] D. H. Douglas and T. K. Peucker, "Algotrithms for the Reduction of the Number of Points Required to Represent a Dig- 


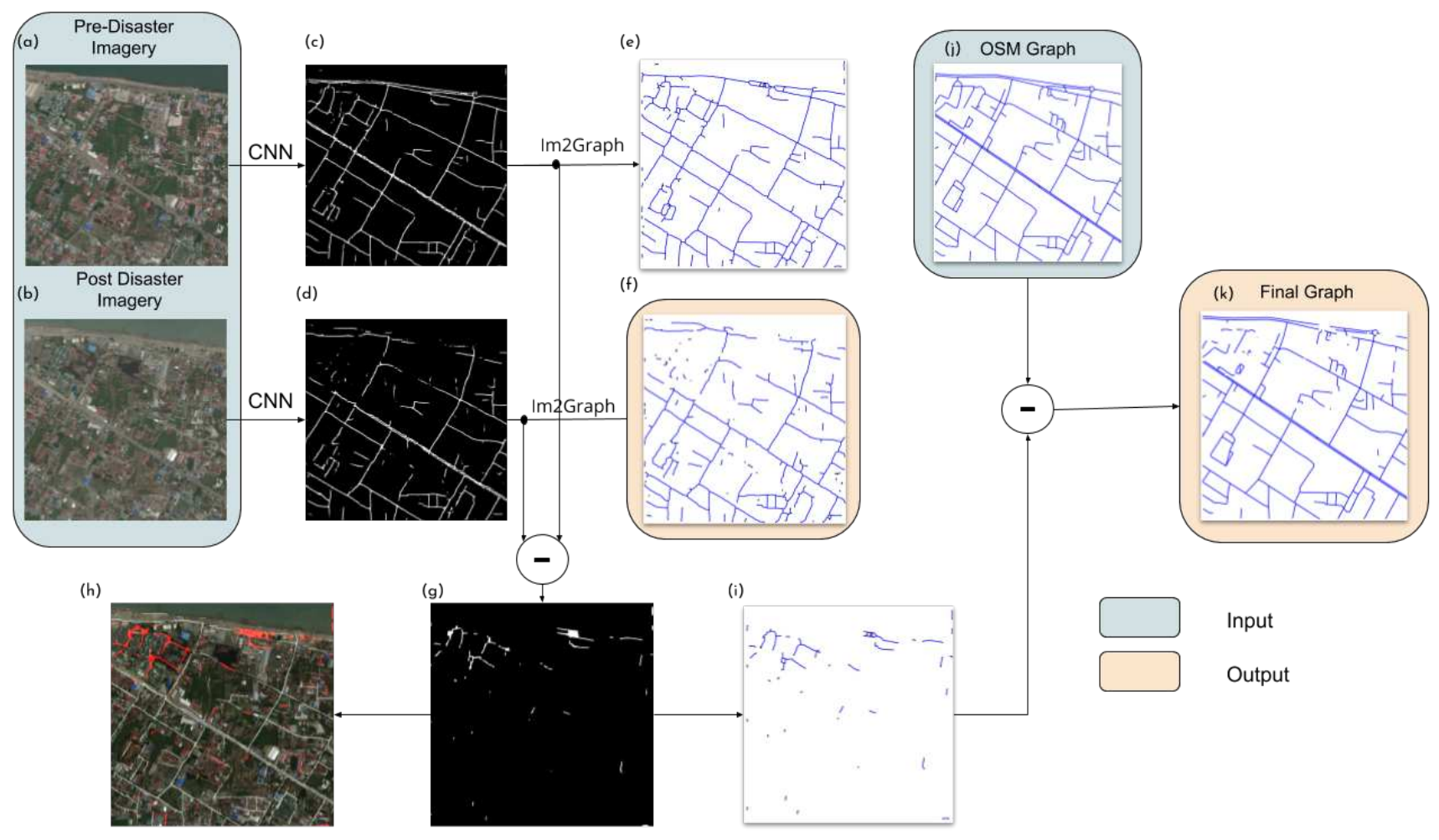

Figure 1: Block Diagram of Proposed Methodology: Pre-disaster satellite image, $(a)$, and post-disaster satellite image, $(b)$, are converted to road masks $(c)$ and $(d)$ respectively using segmentation model. These are then converted to network graphs $(e)$ and $(f)$. The difference in mask images, $(g)$, is converted to a network graph, $(i)$, which is then subtracted from the road network graph taken from OpenStreetMap $(j)$, to produce the final post disaster road network graph, $(k)$. Visualisation of mask difference in shown in $(h)$ where the post-disaster mask is overlaid on the post-disaster image in gray and the difference mask is overlaid in red.
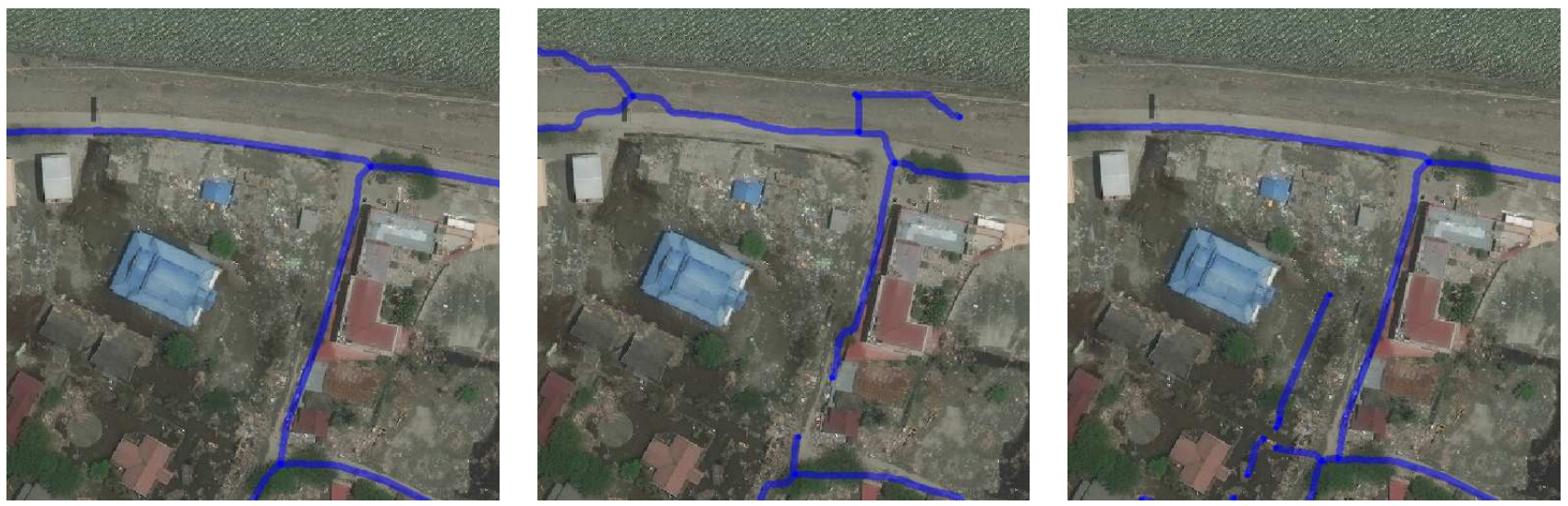

Figure 2: Visualisation of results with extracted roads shown in blue. Left: GT. Middle: DRM[6]. Right: Ours

itized Line or its Caricature," Cartographica: The International Journal for Geographic Information and Geovisualization, vol. 10, pp. 112-122, 121973. 